\title{
Enamel Pits in Hamster Molars, Formed by a Single High Fluoride Dose, Are Associated with a Perturbation of Transitional Stage Ameloblasts
}

\author{
D.M. Lyaruu ${ }^{a} \quad$ L. Vermeulen $^{a} \quad$ N. Stienen ${ }^{a} \quad$ T.J.M. Bervoets ${ }^{a} \quad$ P.K. DenBesten ${ }^{b}$
}

\section{A.L.J.J. Bronckers ${ }^{a}$}

a Department of Oral Cell Biology, ACTA, University of Amsterdam and VU University of Amsterdam, MOVE-Research Institute, Amsterdam, The Netherlands; ${ }^{b}$ Department of Orofacial Sciences, School of Dentistry, University of California at San Francisco, San Francisco, Calif., USA

\section{Key Words}

Cysts • Enamel fluorosis · Histomorphometry • Pits • White spots

\begin{abstract}
Excessive intake of fluoride (F) by young children results in the formation of enamel subsurface porosities and pits, called enamel fluorosis. In this study, we used a single high dose of $\mathrm{F}$ administered to hamster pups to determine the stage of ameloblasts most affected by $\mathrm{F}$ and whether pit formation was related to F-related sub-ameloblastic cyst formation. Hamster pups received a single subcutaneous injection of either $20 \mathrm{mg}$ or $40 \mathrm{mg} \mathrm{NaF} / \mathrm{kg}$ body weight, were sacrificed $24 \mathrm{~h}$ later, and the number of cysts formed in the first molars were counted. Other pups were sacrificed 8 days after $\mathrm{F}$ injection, when the first molars had just erupted, to score for enamel defects. All F-injected pups formed enamel defects in the upper half of the cusps in a dose-dependent way. After injection of $20 \mathrm{mg} \mathrm{NaF} / \mathrm{kg}$, an average of 2.5 white spots per molar was found but no pits. At $40 \mathrm{mg} \mathrm{NaF} / \mathrm{kg}$, almost 4.5 spots per molar were counted as well as 2 pits per molar. The defects in erupted enamel were located in the upper half of the cusps, sites where cysts had formed at the
\end{abstract}

transition stage of ameloblast differentiation. These results suggest that transitional ameloblasts, located between secretory- and maturation-stage ameloblasts, are most sensitive to the effects of a single high dose of F. F-induced cysts formed earlier at the pre-secretory stage were not correlated to either white spots or enamel pits, suggesting that damaged ameloblasts overlying a F-induced cyst regenerate and continue to form enamel.

Copyright $\odot 2012$ S. Karger AG, Basel

Excessive intake of fluoride (F) during dental development results in the formation of mineralization defects in the enamel [Fejerskov et al., 1977, 1994; Ishii and Suckling, 1986; DenBesten, 1999; Hong et al., 2006; DenBesten and Li, 2011]. These changes vary from formation of white opaque lines and small chalk-white areas consisting of subsurface porosities to extensive surface depressions, grooves and multiple pits. How these more severe defects are formed is unclear. Some investigators have suggested that the formation of hypoplastic pits is due to the loss of late secretory ameloblast function [Kierdorf et al., 2004]. Others have hypothesized that pit formation is a post-eruptive event resulting from me-

\section{KARGER}

Fax +41613061234

E-Mail karger@karger.ch

www.karger.com
(C) 2012 S. Karger AG, Basel

0008-6568/12/0466-0575\$38.00/0

Accessible online at:

www.karger.com/cre
A. Bronckers

Oral Cell Biology, ACTA, Vrije Universiteit

Gustav Mahlerlaan 3004

NL-1081 LA Amsterdam (The Netherlands)

Tel. +31 20598 0229, E-Mail a.bronckers@ acta.nl 
chanical damage of the intact surface layer, followed by erosion of the porous sub-subsurface layer when teeth become functional [Thylstrup and Fejerskov, 1978; Fejerskov et al., 1994].

Numerous studies, including ours, have shown that a single $\mathrm{F}$ dose injected into rodents induce dose-dependent changes in the enamel organ and forming enamel [Kruger, 1970; Walton and Eisenman, 1974; Mornstad and Hammarström, 1978; Lyaruu et al., 1989; review: Bronckers et al., 2009]. At 10-20 mg NaF/kg body weight (b.w.), ameloblasts can detach from the enamel surface and form sub-ameloblastic cysts on top of the forming enamel [Mornstad and Hammarström, 1978; Nordlund et al., 1986; Nordlund and Lindskog, 1986; Lyaruu et al., 1989, 2006, 2011; Prostak and Skobe, 1996]. A higher F dose of $60 \mathrm{mg} \mathrm{NaF} / \mathrm{kg}$ results in the entire layer of secretory ameloblasts detaching from the enamel surface [Nordlund et al., 1986].

We found that when hamster pups were injected with $20 \mathrm{mg} \mathrm{NaF} / \mathrm{kg}$ b.w., cysts form preferentially under both early and late secretory ameloblasts [Lyaruu et al., 2006, 2011]. Sub-ameloblastic cysts, including a detached but intact ameloblast layer, developed as early as $1 \mathrm{~h}$ after injection and reached an average number of almost 4 cysts formed per molar at $16 \mathrm{~h}$. However, all sub-ameloblastic cysts disappeared by day 3 after injection [Lyaruu et al., 2011], and newly formed ameloblasts overlying the enamel surface where the cysts had formed were undistinguishable from normal ameloblasts. When the molars were allowed to erupt, 8 days after injection, white spots were present on the enamel surface at the location where cysts formed under late secretory ameloblasts. However, the enamel surface of the white spots was intact and depressions or pits were not found.

In the present study, we tested the hypothesis that pit formation can occur pre-eruptively at higher doses of F. We used the hamster molar model to examine how many cysts were formed before eruption and how many white spots and pits could be seen just after eruption of the teeth into the oral cavity. We correlated the location of the enamel surface to the stage of ameloblast formation when $\mathrm{F}$ was administered, to determine the stage of ameloblasts most sensitive to acute $\mathrm{F}$ exposure.

\section{Materials and Methods}

\section{Animals and Tissues}

Fourteen hamster pups, 4 days old ( $4-5$ g b.w.), were divided into two groups consisting of 7 pups each. The first group received a single subcutaneous injection of $20 \mathrm{mg} \mathrm{NaF} / \mathrm{kg}$ b.w. ( $9 \mathrm{mg} \mathrm{F} / \mathrm{kg}$ b.w.) dissolved in $20 \mu$ l distilled water per gram body weight ( $\mathrm{n}=$ 4) or equimolar $\mathrm{NaCl}(\mathrm{n}=3)$ serving as controls. The second group received a dose of $40 \mathrm{mg} \mathrm{NaF} / \mathrm{kg}(18 \mathrm{mg} \mathrm{F} / \mathrm{kg}$ b.w.) $(\mathrm{n}=4)$ or $\mathrm{NaCl}$ in control pups $(n=3)$. After injection, pups were returned to their mothers. One day after injection, the pups were sacrificed, and jaws were dissected out and fixed for $24 \mathrm{~h}$ in $5 \%$ paraformaldehyde in $0.1 \mathrm{M}$ phosphate buffer $\mathrm{pH} 7.4$ and $2 \%$ sucrose.

In a separate experiment that aimed to examine the effect of cysts on the final structure of enamel, 14 pups were injected with F (20 or $40 \mathrm{mg} \mathrm{NaF/kg} \mathrm{b.w.,} \mathrm{each} \mathrm{n}=4)$ or an equimolar amount of $\mathrm{Cl}^{-}(\mathrm{NaCl}, \mathrm{n}=3$, each group) at day 4 and were returned to their respective mothers for 8 days to allow eruption of the first molars into the oral cavity. All procedures were approved and performed by the Animal Care Committee of the Vrije Universiteit in accordance with national guidelines.

\section{Histological Processing}

A total of 3 hemi-mandibles from different pups injected with $20 \mathrm{mg} \mathrm{NaF} / \mathrm{kg}$ b.w. and 3 from control pups $(\mathrm{NaCl})$ were dehydrated in ethanol and embedded undecalcified in Historesin ${ }^{\circledR}$ (Leica, Microsystems, Mannheim, Germany). Serial sagittal sections, $5 \mu \mathrm{m}$ thick, through the entire first and second molars were made with stainless steel knives using a Reichert-Jung K (Leica, Microsystems) heavy duty microtome. An average of 80 glass slides was collected per hemi-mandible, with each slide containing 4-6 tissue sections. Any missing sections were recorded and accounted for. Every 10th slide was stained with hematoxylineosin $(\mathrm{HE})$, and every 11 th with toluidine blue in $(0.1 \%)$ borax.

\section{Histomorphometry of Sub-Ameloblastic Cysts in Pre-Eruptive} Stages

HE-stained sections (every 10th slide) were analyzed to determine the number and approximate position of each cyst. For a complete three-dimensional outline of the cysts, more sections in the area of interest were then stained (every 2 nd or 3 rd glass slide) until the entire cyst was serially imaged. The relative distribution of cysts in each molar was examined by recording the number of cysts in 3 selected areas: mesial, central and distal cusps (fig. 1).

Scoring of White Spots and Pits in Post-Eruptive Stages

Teeth were collected at postnatal day 12 , immediately after eruption of the first molars while pups were still weaning (day 8 after injection), so as to minimize the chance that pits could be formed post-eruptively in hypomineralized areas with intact surfaces. Mandibles and maxillae were fixed as described above and stored in $70 \%$ ethanol. The mucosa of one hemi-mandible and one hemi-maxilla was removed as much as possible, and the molars were cleaned with special care not to damage the surface and then examined at $10 \times$ magnification under a stereomicroscope.

Defects in enamel of the first molars from one hemi-mandible and one hemi-maxilla were examined in two ways. First, a visual examination was performed under white light using a stereomicroscope (Nikon SMZ-10) where changes in smoothness and texture of the enamel surface were examined at $10 \times$ magnification. The molars were first scored moist to locate any surface irregularities, pits, depressions or white spots. The molars were then air-dried with compressed air and scored again. Second, the molars were examined by quantitative light-induced fluorescence (QLF, Clinical Inspector Research Systems BV) using monochromatic blue light of $404 \mathrm{~nm}$ wavelength [Pretty et al., 2006]. Micro- 
graphs were taken with a Zeiss Stemi SV6 microscope at $32 \times$ magnification. Post-eruptive changes in the enamel surface were scored separately (see fig 1a, c), including the mesial, central and distal cuspal areas, at either the anterior, posterior, labial or lingual sides of the teeth.

\section{Calculations and Statistics}

The data were calculated for each pup, per molar or per surface area, and averaged. The averaged data were used to calculate means and standard deviation (SD) for each treatment group. Student's t test (two-tailed, unpaired), rank sum test and ANOVA were used to calculate statistical significance between the control and experimental groups at $\mathrm{p}<0.05$.

\section{Results}

There were no significant differences between the body weights of $\mathrm{NaF}$-injected and $\mathrm{NaCl}$-injected pups at the end of the experiments. All pups, injected at postnatal day 4 with $\mathrm{NaF}$ (either 20 or $40 \mathrm{mg} / \mathrm{kg}$ b.w.) and sacrificed 1 day later for histological examination, had first molars with sub-ameloblastic cysts formed by groups of ameloblasts in the early secretory (lower half of the cusps) or late secretory stage (upper half of the cusps) of enamel formation. In the cyst areas, ameloblasts were detached from the enamel surface but still formed an intact layer (fig. 1b). The enamel surface below the cysts was always highly hypermineralized. No cysts were found in any of the pups injected with $\mathrm{NaCl}$.

We compared the scoring methods used to detect white spots (wet or dry, by conventional visual inspection using white light) (fig. 2). We found that when tooth surfaces were examined wet, no white spots were visualized. However, when tooth surfaces were scored dry, chalkywhite areas became apparent in the $\mathrm{F}$ groups. Under white light, a total of 56 white spots was counted (20 and $40 \mathrm{mg}$ F/kg b.w. combined). Similar values (62 white spots) were scored using QLF, and there were no statistically significant differences between scoring using white light or blue laser light. Analyses using white light were simpler to complete and, therefore, we used visual inspection with white light under dry conditions for the remainder of the analyses. Visual inspection of the newly erupted first molars 8 days after injection showed that white spots were present in the molars of all F-injected pups $(\mathrm{n}=8)$. Molars of pups receiving $\mathrm{NaCl}(\mathrm{n}=6)$ had no white spots.

In pups that received $20 \mathrm{mg} \mathrm{NaF} / \mathrm{kg}$ b.w. and were examined 8 days later, a total of 21 white spots was counted in the newly erupted first molars, with no significant difference between upper and lower molars. The average
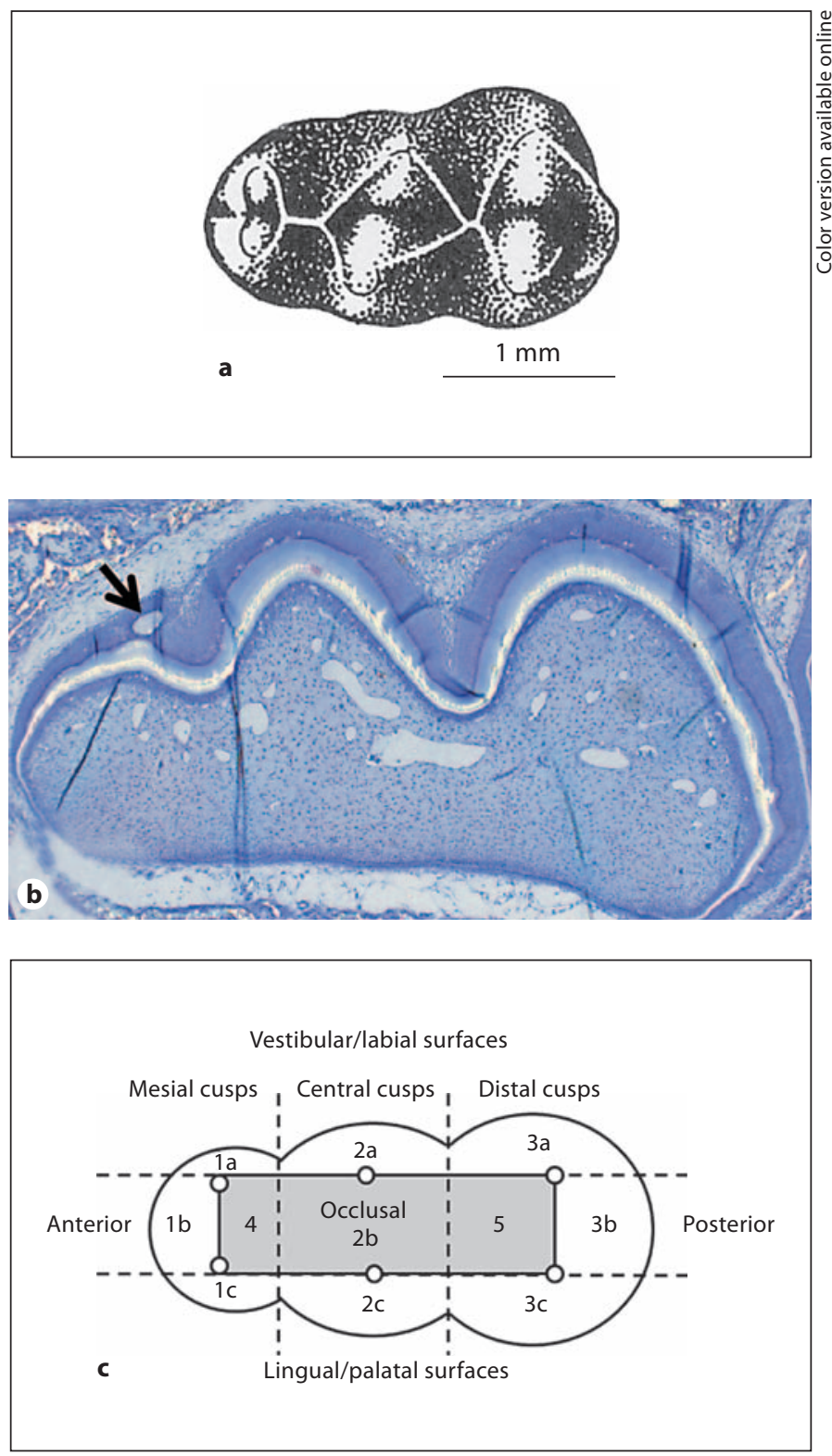

Fig. 1. a Erupted first mandibular molar of the hamster, top (occlusal) view. Hamster first molars have 6 upright cusps in 3 successive rows of two (either labial or lingual), including a mesial, central and distal pair. (Drawing adapted from Gaunt [1961] with permission.) b Sagittal section of an unerupted mandibular molar $24 \mathrm{~h}$ after a single injection of $20 \mathrm{mg} \mathrm{NaF} / \mathrm{kg}$ b.w. Arrow indicates cyst. Toluidine blue staining, $\times 25$. c Schematic representation of the various tooth surfaces screened for enamel defects. The grey area indicates the occlusal surface, defined as the area within the lines that connect the cusp tips (small circles). Vestibular surfaces: $1 a+2 a+3 a$; lingual surfaces: $1 c+2 c+3 c$; anterior (mesial) surface: $1 b$; posterior (distal) surface: $3 b$; mesial cusps: $1 a+1 b+1 c+4$; central cusps: $2 \mathrm{a}+2 \mathrm{~b}+2 \mathrm{c}$; distal cusps: $3 \mathrm{a}+3 \mathrm{~b}+3 \mathrm{c}+5$; occlusal surfaces: $2 \mathrm{~b}+4+5$. 

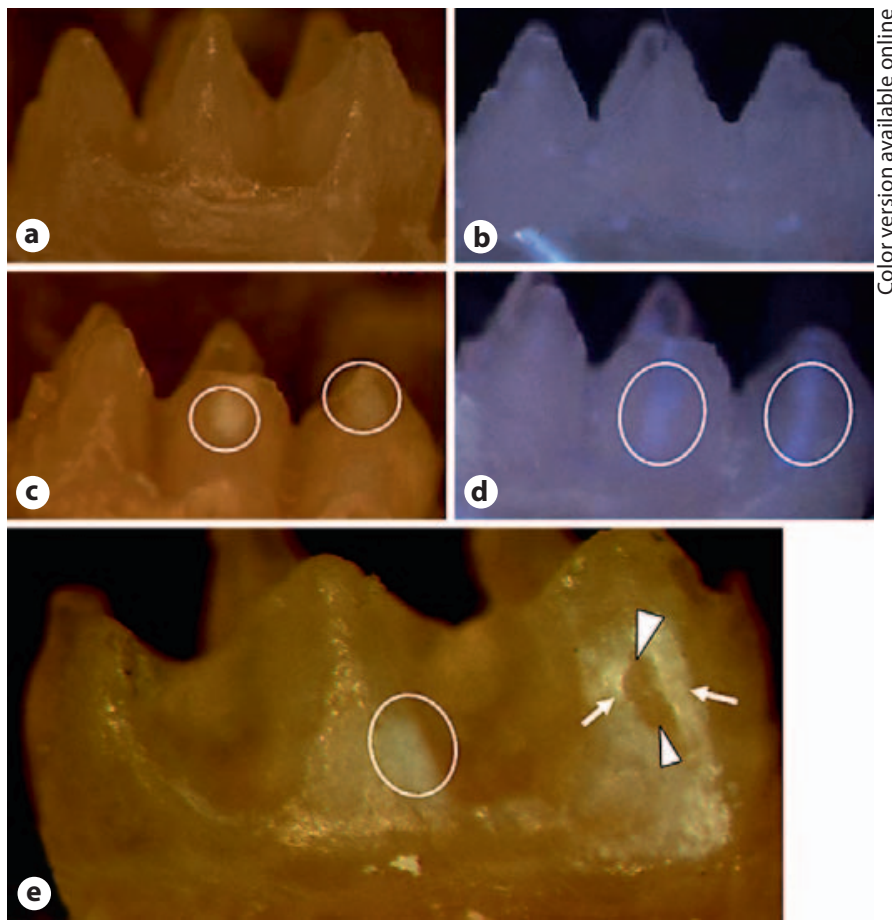

Fig. 2. a-e Erupted first molar from 12-day-old postnatal pups, 8 days after injection of F. a, b Control molar injected with $\mathrm{NaCl}$ seen under either white light (a) or laser light (QLF; b). c, d Molar of a pup injected with $20 \mathrm{mg} \mathrm{NaF} / \mathrm{kg}$ b.w. seen under either white light (c) or laser light (QLF, d). e Molar of a pup injected with $40 \mathrm{mg} \mathrm{NaF} / \mathrm{kg}$ b.w. under white light. White spots are located within white circles (b, c), and in e, a large pit is indicated by white arrowheads. Note that around the pit defect, the enamel surface is opaque white (white arrows).

number of spots per molar was 2.5 (SD: 0.6) for upper molars and 2.7 (SD: 0.9) for lower molars. After injection of $40 \mathrm{mg} \mathrm{NaF} / \mathrm{kg}$ b.w., a total of 35 white spots was counted, and the average number of white spots per molar increased to 4.7 (SD: 2.5) for upper molars and 4.0 (SD: 1.1) for lower molars.

Enamel pits did not form at the lower dose of $\mathrm{F}$. At the higher dose of $\mathrm{F}$, however, an average of almost 2 pits per molar was recorded (mean 1.9 pits per molar, SD: 1.5; $\mathrm{p}<0.05$ ), with no difference between upper and lower first molars.

We then determined whether the number of white spots seen 8 days after injection with $20 \mathrm{mg} \mathrm{NaF} / \mathrm{kg}$ b.w. corresponded to the number of cysts seen at that location in histological sections $24 \mathrm{~h}$ after injection. Mandibular first molars of pups injected with $20 \mathrm{mg} \mathrm{NaF} / \mathrm{kg}$ b.w. formed 1.3 (SD: 0.6) cysts per molar in the lower half of the cusps, below early secretory ameloblasts at

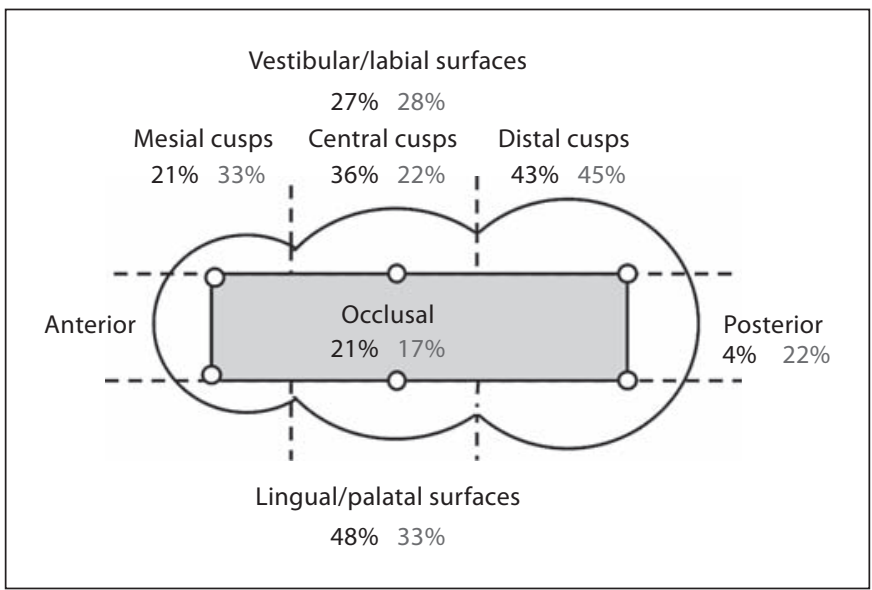

Fig. 3. The distribution of white spots (percentages in black) and pits (percentages in grey) over the various enamel surfaces show a preference for spots to form in lingual/palatal surfaces as compared to vestibular/labial surfaces $(\mathrm{p}<0.05)$ and in the pair of distal cusps compared to mesial cusps $(\mathrm{p}<0.05)$. The calculations were made from the collective data from upper and lower molars, treated with 20 and $40 \mathrm{mg} \mathrm{NaF} / \mathrm{kg}$ b.w. Cumulative number of white spots: $n=56$. Cumulative number of pits: $n=15$.

the cervical loop area, and 1.7 (SD: 2.8) cysts per molar were found below transitional ameloblasts in the upper half of the cusps. Immediately after eruption, no white spots were found in the lower half of the cusps, but 2.7 (SD: 0.9) white spots per molar were found in the upper half.

Finally, we examined whether the white spots and pits were randomly distributed over the various enamel surfaces or whether they developed in preferential locations (fig. 3). There was a preference to form almost twice as many white spots at the lingual surfaces than at the buccal surfaces $(\mathrm{p}<0.05)$, with few spots at the anterior (mesial) surface (fig. 3). There was also a preference to develop more white spots $(\mathrm{p}<0.05)$ and pits in the distal cuspal pair than in the mesial pair (fig. 3). This effect was seen in particular in the lower first molars, but less so in the upper first molars (data not shown).

\section{Discussion}

Treatment with F did not affect the overall growth, i.e. gain in body weight, of the experimental animals as compared to controls, suggesting a specific effect on enamel formation rather than a systemic effect. Our findings show that a single high dose of $\mathrm{F}$ can induce the formation 
of enamel pits in hamster molars even before the tooth has erupted into the oral cavity. Since the molars were examined soon after eruption when pups were not yet completely weaned, it is unlikely that pits had formed from white spots turning into pits by the collapse of fluorotic subsurface porosities due to mastication on solid food particles.

The dose of $\mathrm{F}$ required to induce pre-eruptive formation of enamel pits was relatively high $(40 \mathrm{mg} \mathrm{NaF} / \mathrm{kg}$ b.w.). In 6-day-old neonatal rats, this $\mathrm{F}$ dose results in a short-lasting plasma peak level of $1.7 \mathrm{~mm} \mathrm{~F}$ [Larsen et al., 1981]. Such high plasma peak levels are highly toxic in man and normally are unlikely to occur. In mini pigs, hypoplastic pit formation was reported to occur at much lower F levels ( 2 or $0.9 \mathrm{mg} \mathrm{F} / \mathrm{kg}$ b.w. given orally in food) over an extended period of time (1 year) [Kierdorf et al., 2004]. In sheep, a daily dose of 0.2 or $0.5 \mathrm{mg} \mathrm{F} / \mathrm{kg}$ b.w. for 6 months induced white opacities, while an oral daily dose of 2 or $6 \mathrm{mg} \mathrm{F} / \mathrm{kg}$ for 21 days caused formation of hypoplastic lesions [Nelson et al., 1989]. These studies show that the formation of enamel opacities or pits secondary to $\mathrm{F}$ exposure is related to the dose and duration of exposure in each particular species.

One interesting finding was that even though $\mathrm{F}$ induced cyst formation in both the early secretory stage and late secretory/transition stage of ameloblast differentiation, white spots only formed under the late secretory ameloblasts. Hence, the cysts in the cervical loop region apparently did not result in the formation of white spots. It is possible that defects in the cervical loop area are repaired by newly differentiated ameloblasts. However, it is also possible that these early formed defects are buried in progressively deeper layers of enamel, resulting in the eventual formation of a sound enamel surface. Later formed defects would remain visible as they occur closer to the final enamel surface layer.

There was a tendency for the upper half of the cusps to develop more white spots than cysts had formed, suggesting that other mechanisms different from cyst formation may also lead to the development of fluorotic white spots. This is consistent with reports that subsurface mineralization defects in incisor enamel can develop without overt cyst formation in rats exposed to fluoridated drinking water or following a single injection of low doses of $\mathrm{F}$ [Angmar-Mansson and Whitford, 1982, 1984; DenBesten et al., 1985; Ishii and Suckling, 1986; Richards et al., 1986]. The location of these additional white spots in the upper half of the cusps indicates an effect at the transition stage of enamel formation, possibly related to changes in the modulation patterns of early maturation ameloblasts
[DenBesten et al., 1985; Richards et al., 1986; Smith et al., 1993].

Fluorotic white spots and pits formed preferentially in the lingual/palatal surfaces. These results are consistent with the observation that, in man, more fluorotic changes are seen in the lingual surfaces of the molars and premolars of the maxilla, while the mandibular teeth show a tendency for more severe fluorotic changes in their buccal surfaces [Thylstrup and Fejerskov, 1978]. The reason for this difference is unknown but may be due to differences in the developmental stage of the ameloblasts at different sites of the cusps. In rodents, morphodifferentiation in lower molars is slightly more advanced than in the upper molars. In addition, in hamster molar tooth germs, the central cusps develop earlier than the distal and mesial ones.

In conclusion, these studies have shown that F-induced sub-ameloblastic cysts formed below late secretory and transitional ameloblasts can result in the formation of fluorotic white spots or subsurface porosities with intact surfaces. Formation of enamel pits (or local hypoplastic enamel) in hamsters before eruption into the oral cavity can occur, but only at high $\mathrm{F}$ doses. These results suggest that pit formation is related to a localized F-related damage to ameloblasts occurring at the transition stage of enamel formation.

\section{Acknowledgments}

The work has been supported in part by NIH grant DE1350806 . The funders had no role in study design, data collection and analysis, decision to publish, or preparation of the manuscript. The authors acknowledge the help of Dr. Moniek van der Veen (Orthodontics, ACTA) who kindly assisted in QLF analyses and Mark Schoonderwoerd for technical assistance.

References

Angmar-Mansson B, Whitford GM: Plasma fluoride levels and enamel fluorosis in the rat. Caries Res 1982;16:334-339.

-Angmar-Mansson B, Whitford GM: Enamel fluorosis related to plasma $\mathrm{F}$ levels in the rat. Caries Res 1984;18:25-32.

Bronckers AL, Lyaruu DM, DenBesten PK: The impact of fluoride on ameloblasts and the mechanisms of enamel fluorosis. J Dent Res 2009;88:877-893.

DenBesten PK, Crenshaw MA, Wilson MH: Changes in the fluoride-induced modulation of maturation stage ameloblasts of rats. J Dent Res 1985;64:1365-1370. 
DenBesten PK: Biological mechanisms of dental fluorosis relevant to the use of fluoride supplements. Community Dent Oral Epidemiol 1999;27:41-47.

DenBesten PK, Li W: Chronic fluoride toxicity: dental fluorosis; in: Buzalaf MAR (ed): Fluoride and the Oral Environment. Monogr Oral Sci. Basel, Karger, 2011, vol 22, pp 8196.

Fejerskov O, Thylstrup A, Larsen MJ: Clinical and structural features and possible pathogenic mechanisms of dental fluorosis. Scand J Dent Res 1977;85:510-534

-Fejerskov O, Larsen MJ, Richards A, Baelum V: Dental tissue effects of fluoride. Adv Dent Res 1994;8:15-31.

Gaunt WA: The development of the molar pattern of the golden hamster (Mesocricetus auratus $\mathrm{W}$.$) , together with a re-assessment of$ the molar pattern of the mouse (Mus musculus). Acta Anat 1961;45:219-254.

-Hong L, Levy SM, Warren JJ, Broffitt B, Cavanaugh J: Fluoride intake levels in relation to fluorosis development in permanent maxillary central incisors and first molars. Caries Res 2006;40:494-500.

Ishii T, Suckling G: The appearance of tooth enamel in children ingesting water with high fluoride content for a limited period during early tooth development. J Dent Res 1986;65: 974-977.
Kierdorf H, Kierdorf U, Richards A, Josephsen K: Fluoride-induced alterations of enamel structure: an experimental study in the miniature pig. Anat Embryol 2004;207:463-474.

Kruger BJ: The effect of different levels of fluoride on the ultra-structure of ameloblasts in the rat. Arch Oral Biol 1970;15:109-114.

Larsen MJ, Richards A, Fejerskov O: Effect of intraperitoneally injected fluoride on plasma calcium in suckling and adult rats. Calcif Tissue Int 1981;33:541-544.

Lyaruu DM, Lenglet WJ, Wöltgens JH, Bronckers AL: Micro-PIGE determination of fluorine distribution in developing hamster tooth germs. J Histochem Cytochem 1989; 37:581-587.

Lyaruu DM, Bervoets TJ, Bronckers AL: Short exposure to high levels of fluoride induces stage-dependent structural changes in ameloblasts and enamel mineralization. Eur J Oral Sci 2006;114(suppl 1):111-115.

Lyaruu DM, Alberga JM, Kwee NC, Bervoets TJ, Bronckers AL, DenBesten PK: Fate of fluoride-induced subameloblastic cysts in developing hamster molar tooth germs. Arch Oral Biol 2011;56:238-243.

Mornstad H, Hammarström L: Morphologic changes in the rat enamel organ following a single intraperitoneal injection of sodium fluoride. Scand J Dent Res 1978;86:211-220.

-Nelson DGA, Copote GE, Vickridge IC, Suckling G: Proton microprobe determination of fluorine profiles in the enamel and dentine of erupting incisors from sheep given low and high daily doses of fluoride. Arch Oral Biol 1989;34:419-429.
Nordlund AL, Ekstrand JL, Hammarström L: Fluoride-induced cystic changes in the enamel organ of the rat molar. J Oral Pathol 1986;15:87-92.

-Nordlund AL, Lindskog S: Scanning electron microscopy of fluoride-induced disturbances on the enamel surface of rat molars. Scand J Dent Res 1986;94:185-192.

Pretty IA, Tavener JA, Browne D, Brettle DS, Whelton H, Ellwood RP: Quantification of dental fluorosis using fluorescence imaging. Caries Res 2006;40:426-434.

Prostak KS, Skobe Z: Anion translocation through the enamel organ. Adv Dent Res 1996;10:238-244.

Richards A, Kragstrup J, Josephsen K, Fejerskov O: Dental fluorosis developed in post-secretory enamel. J Dent Res 1986;65:1406-1409.

- Smith CE, Nanci A, DenBesten PK: Effects of chronic fluoride exposure on morphometric parameters defining the stages of amelogenesis and ameloblast modulation in rat incisors. Anat Rec 1993;237:243-258.

- Thylstrup A, Fejerskov O: Clinical appearance of dental fluorosis in permanent teeth in relation to histologic changes. Community Dent Oral Epidemiol 1978;6:315-328.

Walton RE, Eisenmann DR: Ultrastructural examination of various stages of amelogenesis in the rat following parenteral fluoride administration. Arch Oral Biol 1974;19:171182 . 